\title{
Sub-Poissonian multiplicity distributions in jets produced in hadron collisions
}

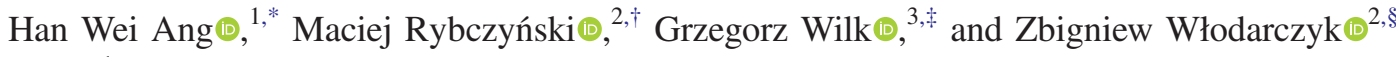 \\ ${ }^{1}$ Department of Physics, National University of Singapore, Singapore 117551, Singapore \\ ${ }^{2}$ Institute of Physics, Jan Kochanowski University, 25-406 Kielce, Poland \\ ${ }^{3}$ National Centre for Nuclear Research, Warsaw 00-681, Poland
}

(Received 17 November 2021; accepted 10 February 2022; published 4 March 2022)

In this work we show that the proper analysis and interpretation of the experimental data on the multiplicity distributions of charged particles produced in jets measured in the ATLAS experiment at the LHC indicates their sub-Poissonian nature. We also show how, by using the recurrence relations and combinants of these distributions, one can obtain new and otherwise unavailable information contained in them, which may broaden our knowledge of the particle production mechanism.

DOI: 10.1103/PhysRevD.105.054003

\section{INTRODUCTION}

The experimentally measured multiplicity distributions $P(N)$ of the produced particles are the main source of information about the dynamics of their production processes $[1,2]$. In the theoretical description they are characterized by the generating functions,

$$
G(z)=\sum_{N=0}^{\infty} P(N) z^{N}
$$

such that

$$
P(N)=\left.\frac{1}{N !} \frac{d^{N} G(z)}{d z^{N}}\right|_{z=0}
$$

Preliminary information about $P(N)$ is provided by the moments of this distribution,

$$
m_{k}=\sum_{N=0}^{\infty}(N-c)^{k} P(N)
$$

In many cases, it is sufficient to analyze only the two lowest moments, namely, the mean value $\langle N\rangle=m_{1}$ [being the first

\footnotetext{
*ang.h.w@u.nus.edu

†maciej.rybczynski@ujk.edu.pl

*grzegorz.wilk@ncbj.gov.pl

§bigniew.wlodarczyk@ujk.edu.pl
}

Published by the American Physical Society under the terms of the Creative Commons Attribution 4.0 International license. Further distribution of this work must maintain attribution to the author(s) and the published article's title, journal citation, and DOI. Funded by SCOAP ${ }^{3}$. raw moment $(c=0)$ ] and variance $\operatorname{Var}(N)=m_{2}$ [being the second central moment $(c=\langle N\rangle)]$.

Another way to characterize $P(N)$ is through a recursive formula,

$$
(N+1) P(N+1)=g(N) P(N),
$$

that connects adjacent values of $P(N)$ for the production of $N$ and $(N+1)$ particles. It is assumed here that every $P(N)$ is determined only by the next lower $P(N-1)$ value. In other words, a relationship with other $P(N-j)$ for $j>1$ is indirect. The final algebraic form of $P(N)$ is determined by the function $g(N)$. In its simplest form, $g(N)$ is assumed to be a linear function of $N$ given by $g(N)=\alpha+\beta N$. This form is enough to define commonly known and widely used distributions like the Poisson distribution (PD) (for which $\beta=0$ ), binomial distribution (BD) (for which $\beta<0$ ), and negative binomial distribution (NBD) (for which $\beta>0$ ). In general, by selecting the appropriate model, the form of $g(N)$ can be chosen in such a way that the corresponding $P(N)$ describes the experimental data (for example, by introducing higher-order terms [3] or by using its more involved forms $[4,5]$ ).

The more promising approach is to use a $g(N)$ that contains information about the interrelationship between the multiplicity $N$ and all smaller multiplicities recursively,

$$
(N+1) P(N+1)=\langle N\rangle \sum_{j=0}^{N} C_{j} P(N-j) .
$$

The memory of that relationship is encoded in coefficients $C_{j}$ called modified combinants. They were introduced and intensively discussed in Refs. [6-13]. By inverting the recursion (5) we obtain an equation that allows us to determine $C_{j}$ from the measured $P(N)$, 
$\langle N\rangle C_{j}=(j+1)\left[\frac{P(j+1)}{P(0)}\right]-\langle N\rangle \sum_{i=0}^{j-1} C_{i}\left[\frac{P(j-i)}{P(0)}\right]$

(provided we have sufficient statistics). Modified combinants are closely related to combinants $C_{j}^{\star}$,

$$
C_{j}=\frac{j+1}{\langle N\rangle} C_{j+1}^{\star},
$$

introduced in Refs. [14-16] by means of the generating functions $G(z)$ as

$$
\langle N\rangle C_{j}^{\star}=\left.\frac{1}{j !} \frac{d^{j+1} \ln G(z)}{d z^{j+1}}\right|_{z=0},
$$

which have been discussed and used in many publications $[1,2,17-21]$.

Modified combinants are complementary to the commonly used factorial moments $F_{q}$ and cumulant factorial moments $K_{q}$ (see Appendix A for details). They differ in that, while $C_{j}$ 's depend only on multiplicities smaller than their rank, $K_{q}$ 's require the knowledge of all $P(N)$ 's and are therefore very sensitive to possible limitations of the available phase space [1,2]. However, both $C_{j}$ and $K_{q}$ share the property of additivity. It turns out that most of the measured multiplicity distributions $P(N)$ give oscillatory combinants $C_{j}$ with increasing index $j$ which arise due to the presence of a $\mathrm{BD}$ component in the measured $P(N)$ [7-12].

Combinants are believed to be best suited for the study of sparsely populated areas of phase space (while cumulants are better suited for the study of densely populated areas) $[1,2]$. This feature makes them a potentially important tool for studying $P(N)$ in jets where the number of produced particles is small (on the order of $\sim 10$ ). However, the ATLAS data [22] do not include $P(0)$, which is crucial for their determination. This means that in our analysis, we must extrapolate the recurrence relation in Eq. (4) to evaluate $P(0)$ and use the combinants only for additional verification of our conclusions.

In the next section we provide details concerning ATLAS data with particular attention to the fact that the measured multiplicity distributions in the jets are clearly sub-Poissonian in character (with details depending on the phase space covered). This observation will be our main point for further discussion and calculations described in Secs. III and IV. Section V summarizes and concludes our work.

\section{MULTIPLICITY DISTRIBUTIONS OF PARTICLES IN ATLAS JETS}

ATLAS data [22] of jets measured in proton-proton collisions at an energy $\sqrt{s}=7 \mathrm{TeV}$ (using a minimum bias trigger) were taken (data used here come from Refs. [23]).
Jets were reconstructed using the anti- $k_{t}$ algorithm applied to charged particles produced in very narrow cones defined by the radius parameter $R=\sqrt{\Delta \eta^{2}+\Delta \phi^{2}}$ (where $\Delta \phi$ and $\Delta \eta$ are the azimuthal angle and pseudorapidity of the hadrons relative to that of the jet, respectively). Here, $\eta=$ $-\ln \tan \theta$ (with $\theta$ being the polar angle), with $R=0.4$ and again at $R=0.6$. Only data with high statistics within acceptable regions of phase space were selected. They were obtained over five different transverse momentum ranges across four rapidity ranges, giving a total of 20 possible combinations for each of the two radius parameters (with radius parameters $R=0.4$ and $R=0.6$, respectively).

So far, the ATLAS data have been carefully analyzed in terms of possible self-similarity between the $p_{T}$ distributions of jets and $p_{T}$ distributions of particles in these jets [24]. Indeed, their detailed analysis clearly indicates the selfsimilarity of the particle distributions in jets and the distributions of the jets themselves, indicative of the existence of a common mechanism behind all of these processes.

Taking advantage of the fact that ATLAS also publishes data for multiplicity distributions of particles produced in observed jets, we extend this analysis to study the nature of these distributions. The first observation is that the multiplicity distributions of the particles in the jets observed in the ATLAS experiment are sub-Poissonian, cf. Fig. 1 where $\operatorname{Var}(N)<\langle N\rangle$. Although the BD is a possible subPoissonian distribution for $g(N)$, the plot in Fig. 2 derived from the recursive relationship in Eq. (4) is nonlinear.

Therefore, this means that one should switch to a scenario inspired by a possible nonlinear form of the recursion $g(N)$, such as

$$
g(N)=(N+1) \frac{P(N+1)}{P(N)}=\frac{\alpha}{(N+1)^{\delta}} .
$$

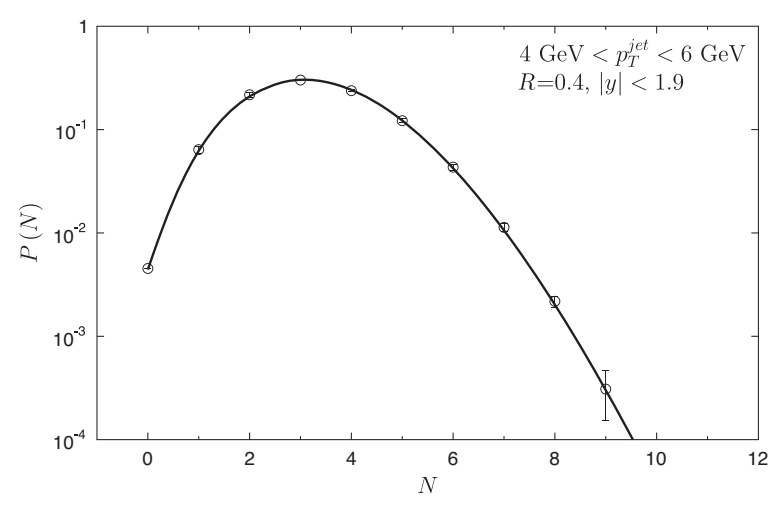

FIG. 1. Multiplicity distribution of charged particles per jet with $4 \mathrm{GeV}<p_{T}^{\text {jet }}<6 \mathrm{GeV}$, over the full measured rapidity range $|y|<1.9$, with radius parameter $R=0.4$. Points show data from the ATLAS experiment [22]. $P(N=0)$ comes from extrapolation of the experimental recurrence relation $g(N)$ to $N=0$. The curve fitting this data comes from Eq. (13) with parameters $\alpha=14.1$ and $\delta=1.07$. 


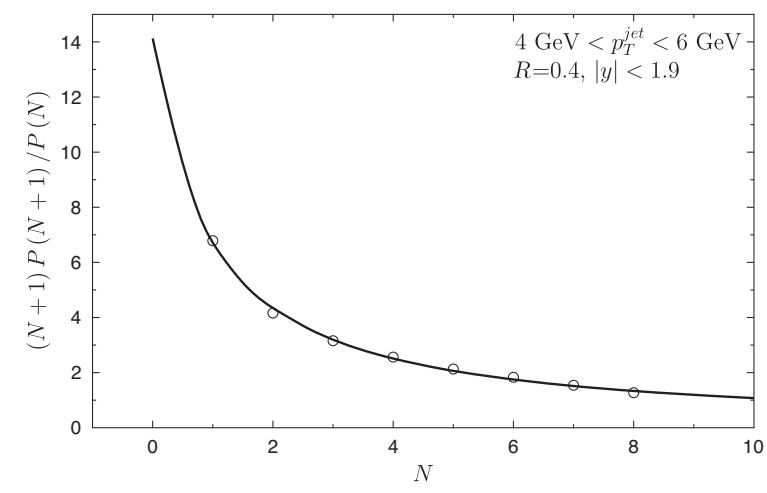

FIG. 2. Recurrent relations. Points show $g(N)$ for the experimental $P(N)$ from Fig. 1. The curve is fit using $g(N)$ from Eq. (9) with $\alpha=14.1$ and $\delta=1.07$.

As shown in Fig. 2, such a form with $\delta=1.07$ fits the data very well. Therefore, we note that for

$$
(N+1) \frac{P(N+1)}{P(N)}=\alpha
$$

we have the PD, with

$P(N)=c \frac{\alpha^{N}}{N !} \quad$ where $\alpha=\langle N\rangle, \quad c=\exp (-\alpha)$.

If we change this recursive relationship to a nonlinear one given by

$$
(N+1)^{1+\delta} \frac{P(N+1)}{P(N)}=\alpha,
$$

we get a sub-Poissonian distribution,

$$
P(N)=c \frac{\alpha^{N}}{(N !)^{1+\delta}}
$$

where $c=P(0)$ is a normalization factor. In Fig. 1 we present a comparison of this multiplicity distribution with the experimental data from ATLAS.

Once we know $P(0)$ from the extrapolation of the experimental recurrence relation $g(N)$ to $N=0$, we are able to determine the corresponding modified combinants from the measured multiplicity distributions $P(N)$ using Eq. (6) [which is important because in ATLAS, the $P(0)$ data points are not directly measured]. The $C_{j}$ 's derived in this way are plotted in Fig. 3 as red circles. They can now be compared with the combinants obtained in the same way but from the theoretical sub-Poissonian distribution $P(N)$ given by Eq. (13). The corresponding results are plotted in Fig. 3 as black squares. We observe characteristic oscillatory behavior of modified combinants with only roughly consistent amplitude. Despite the nice agreement between the multiplicity distributions (shown in Fig. 1), the mentioned combinants indicate a difference between the fit and experimental data.

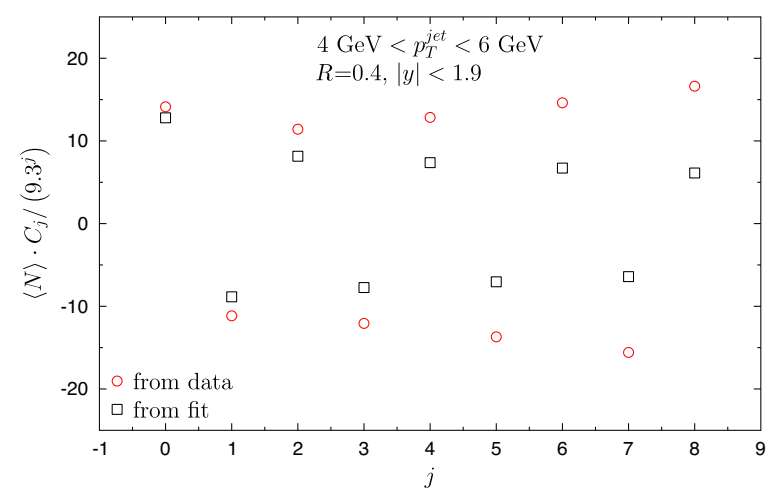

FIG. 3. Comparison of $C_{j}$ for the multiplicity distributions from Fig. 1. Red circles: $C_{j}$ from data on $P(N)$ from Fig. 1. Black squares: from the theoretical $P(N)$ defined by Eq. (13).

In the event where $\delta=1$, Eq. (13) can be written in a closed form given by

$$
P(N)=\frac{1}{I_{0}(2 \sqrt{\alpha})} \frac{\alpha^{N}}{(N !)^{2}},
$$

where

$$
\alpha=\left\langle N^{2}\right\rangle \quad \text { and } \quad c=P(0)=\frac{1}{I_{0}(2 \sqrt{\alpha})},
$$

with $I_{0}$ being the Bessel function of the first kind. In this case $(\delta=1)$, the theoretical $C_{j}$ 's are given by the recurrence relation

$$
\langle N\rangle C_{j}=\frac{(j+1) \alpha^{j+1}}{[(j+1) !]^{2}}-\langle N\rangle \sum_{i=0}^{j-i} C_{i} \frac{\alpha^{j-i}}{[(j-i) !]^{2}},
$$

and are of the form

$$
\langle N\rangle C_{j}=(-1)^{j} \beta_{j} \alpha^{j+1},
$$

where the numbers $\beta_{j}$ are rational: $\beta_{0}=1, \beta_{1}=\frac{1}{2}, \beta_{2}=\frac{1}{3}$, $\beta_{3}=\frac{11}{48}, \beta_{4}=\frac{19}{120}, \beta_{5}=\frac{473}{4320}, \ldots$ They were first calculated by Euler in relation to the positive zeros $\gamma_{l}$ of the Bessel function $J_{0}(z)$ as [25]

$$
\beta_{j+1}=\sum_{l=1}^{\infty}\left(\frac{2}{\gamma_{l}}\right)^{2(j+l)}, \quad j=0,1,2, \ldots
$$

For $j \geq 1$, the coefficients $\beta_{j}$ can be approximated by

$$
\beta_{j} \cong \exp \left[-\frac{j+1}{e}\right] \text {. }
$$

At this point, it is worth noting that $C_{j}$ can help in the search for the correct $P(N)$. Based on our experience thus far, let us assume that strongly oscillating $C_{j}$ (especially 
with a period equal to 2, as seen in Fig. 3) indicate the presence of a single-component $\mathrm{BD}$ in some form. On closer inspection, it is clear that this cannot be the case since the amplitude of oscillations of $C_{j}$ in Fig. 3 grows approximately as $9.3^{j}$ with $j$. Should these $C_{j}$ originate from a single-component $\mathrm{BD}$ with amplitudes given by $[p /(1-p)]^{j}$, it would mean that $p>0.8$. In addition, to reproduce $\langle N\rangle=K p \sim 3.3$ as observed in data, one would require $K<5$. Taken together, these would limit us to multiplicities of $N<5$. This is in contradiction with the measured $P(N)$ where the observed multiplicities $N=5$. By continuing to stick to $\mathrm{BD}$, our previous experiences $[7,9-11]$ tell us that a potential solution might be to use the sum of two BDs instead of one. However, as we will show in Appendix B, it is not possible to describe both $P(N)$ and its corresponding $C_{j}$ with this approach.

Continuing the approach based on $g(N)$, it turns out that with the increase in $p_{T}$ of jets (corresponding to an increase of $\langle N\rangle$ in our case), we observe a deviation from the form of $g(N)$ given in Eq. (9). The modified $g(N)$ can be made to describe data if expressed as a recursive relation given by (see Fig. 4)

$$
g(N)=(N+1) \frac{P(N+1)}{P(N)}=\frac{\alpha}{(N+1)^{\delta}}+\alpha_{0},
$$

leading to the multiplicity distribution (see Fig. 5)

$$
P(N)=\frac{c}{N !} \prod_{i=1}^{N}\left(\frac{\alpha}{i^{\delta}}+\alpha_{0}\right)
$$

with $c=P(0)$. The corresponding $C_{j}$ are shown in Fig. 6 .

Note that for integer parameter $\delta$, Eq. (21) has a closed analytical form, namely, for $\delta=1$

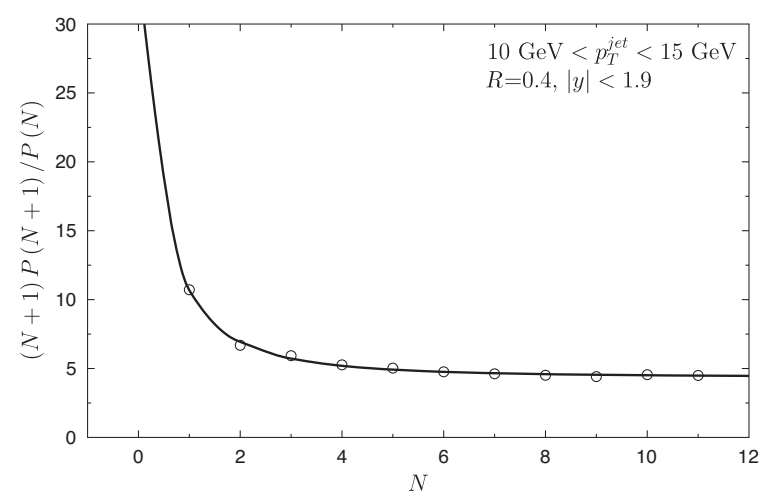

FIG. 4. Recurrent relations $g(N)$ for multiplicity distributions $P(N)$ over the full measured rapidity range $|y|<1.9$, in jets with radius parameter $R=0.4$ and transverse momentum range $10 \mathrm{GeV}<p_{T}^{\text {jet }}<15 \mathrm{GeV}$. Points: $g(N)$ from experimental data [22]. Curve: fit using $g(N)$ from Eq. (20) with parameters $\alpha=29.4, \delta=2.20$, and $\alpha_{0}=4.37$.

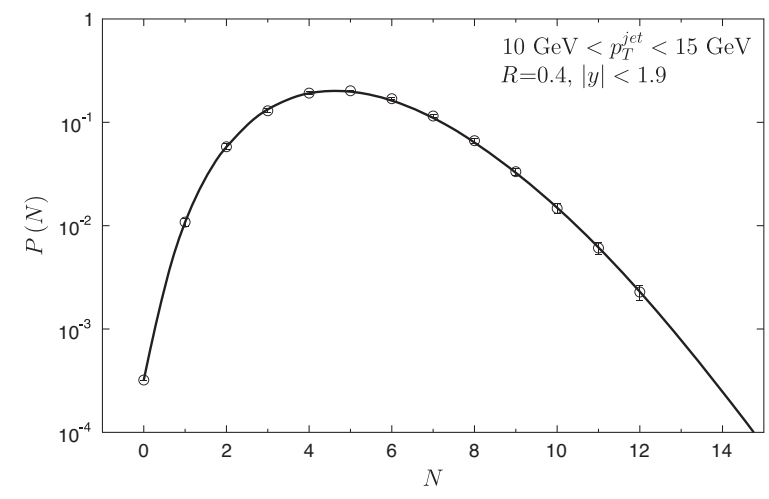

FIG. 5. Points: $P(N)$ from ATLAS data for jets with $10 \mathrm{GeV}<$ $p_{T}^{\text {jet }}<15 \mathrm{GeV}$ and radius parameter $R=04$, over the full measured rapidity range $|y|<1.9$. The curve fitting these data comes from Eq. (21) with parameters: $c=3.2 \times 10^{-4}, \alpha=29.4$, $\alpha_{0}=4.37$ and $\delta=2.20$.

$$
P(N)=P(0) \frac{\alpha_{0}^{N}}{(N !)^{2}} \frac{\Gamma\left(1+\frac{\alpha}{\alpha_{0}}+N\right)}{\Gamma\left(1+\frac{\alpha}{\alpha_{0}}\right)},
$$

while for $\delta=2$

$$
\begin{aligned}
P(N)= & P(0) \frac{\alpha_{0}^{N}}{(N !)^{3}} . \\
& \cdot \frac{\Gamma\left(1-\sqrt{\frac{-\alpha}{\alpha_{0}}}+N\right)}{\Gamma\left(1-\sqrt{\frac{-\alpha}{\alpha_{0}}}\right)} \frac{\Gamma\left(1+\sqrt{\frac{-\alpha}{\alpha_{0}}}+N\right)}{\Gamma\left(1+\sqrt{\frac{-\alpha}{\alpha_{0}}}\right)},
\end{aligned}
$$

and for higher values of $\delta$ we have the product of $\delta$ Pochhammer symbols,

$$
\left(x_{l}\right)_{N}=\frac{\Gamma\left(x_{i}+N\right)}{\Gamma\left(x_{i}\right)},
$$

where $x_{l}=1+(-1)^{l}(-1)^{l / \delta}\left(\alpha / \alpha_{0}\right)^{1 / \delta}$ for $l=0,1, \ldots, \delta-1$.

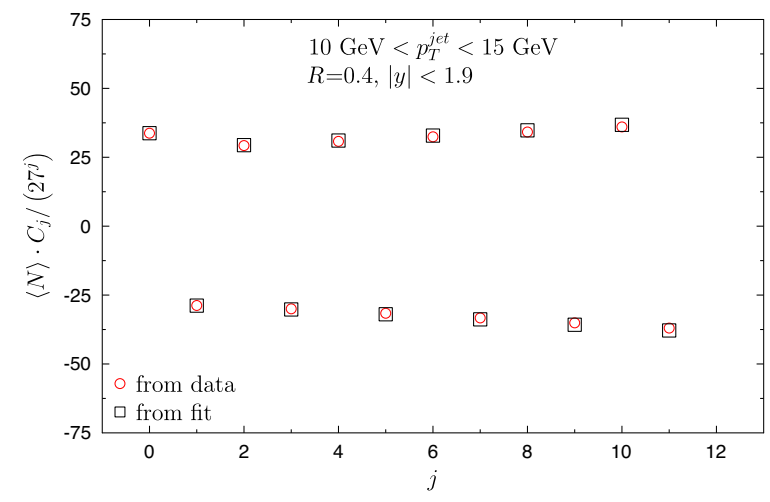

FIG. 6. Comparison of $C_{j}$ for multiplicity distributions in jets. Red circles: $C_{j}$ from data on $P(N)$; black squares: from theoretical $P(N)$ defined by Eq. (21). Parameters are the same as in Fig. 5. 


\section{POSSIBLE EXPLANATION: MULTIPLICITY- DEPENDENT BIRTH AND DEATH RATES}

To interpret the results shown in the previous section, note that Eq. (13) actually represents the so-called COMPoisson distribution introduced by Conway and Maxwell [26] as a model for steady-state queuing systems with statedependent arrival or service rates (in other words, a birthdeath process with Poisson arrival rate and exponential service rate). It was rediscovered in Ref. [27] where the term Conway-Maxwell-Poisson was proposed, and a detailed study of its properties and applications was performed. More recent studies can be found in Refs. $[28,29]$. To the best of our knowledge, this distribution has not been used in the analysis of multiplicity distributions of particles produced in multiparticle production processes.

We will now show that the form of the COM-Poissonian distribution can be obtained from a stochastic Markov process with multiplicity-dependent birth and death rates denoted by $\lambda_{N}$ and $\mu_{N}$, respectively [30]. Let $P(N, t)$ be the probability of having $N$ particles at time $t$ and let us consider a very general birth-death process given by the following equations:

$$
\begin{aligned}
& P^{\prime}(0, t)=-\lambda_{0} P(0, t)+\mu_{1} P(1, t), \\
& P^{\prime}(N, t)=-\left(\lambda_{N}+\mu_{N}\right) P(N, t) \\
&+\lambda_{N-1} P(N-1, r)+\mu_{N+1} P(N+1, t) .
\end{aligned}
$$

If we assume the forms

$$
\lambda_{N}=\frac{\lambda}{(N+1)^{a}} \quad \text { and } \quad \mu_{N}=N^{b} \mu
$$

we get

$$
\begin{aligned}
- & {\left[\frac{\lambda}{(N+1)^{a}}+N^{b} \mu\right] P(N) } \\
& +\frac{\lambda}{N^{a}} P(N-1)+(N+1)^{b} \mu P(N+1)=0
\end{aligned}
$$

for the steady state, where $P^{\prime}(N, t)=0$. If we denote

$$
\frac{\lambda}{\mu}=\alpha,
$$

we can rewrite Eq. (28) as

$$
\begin{aligned}
- & \frac{\alpha}{(N+1)^{a}} P(N)-N^{b} P(N) \\
& +\frac{\alpha}{N^{a}} P(N-1)+(N+1)^{b} P(N+1)=0,
\end{aligned}
$$

which leads to the recurrence relation

$$
(N+1)^{b} P(N+1)=\frac{\alpha}{(N+1)^{a}} P(N) .
$$

Further simplifications can be made by writing

$$
a+b=\nu,
$$

which gives us

$$
\frac{P(N+1)}{P(N)}=\frac{\alpha}{(N+1)^{\nu}} .
$$

For $\delta=\nu-1$, this is just the recurrence form of the COMPoisson distribution defined by Eq. (12). The condition in Eq. (32) has allowed us to successfully reparametrize the recurrence relation of Eq. (33) using $\alpha$ and $\nu=a+b$. This corresponds to the entire class of Markov processes previously characterized by the parameters $a$ and $b$ with the birth and death rates given in Eq. (27).

However, to describe the distribution defined by Eqs. (20) and (21) (which is no longer the COMPoisson distribution) while still using the birth-death process, we need to add an additional term $\lambda^{\star}$ to the birth rate $\lambda_{N}$,

$$
\lambda_{N}=\frac{\lambda}{(N+1)^{a}}+\lambda^{\star}(N+1)^{(b-1)} .
$$

If we substitute Eq. (34) into Eq. (26) and denote

$$
\frac{\lambda^{\star}}{\mu}=\alpha_{0},
$$

we get the recurrence relation

$$
\frac{P(N+1)}{P(N)}=\frac{\alpha}{(N+1)^{\nu}}+\frac{\alpha_{0}}{N+1},
$$

corresponding to Eq. (20).

\section{SUMMARY OF RESULTS}

The ATLAS data suitable for our purposes cover five different ranges of transverse momentum, each further divided into four different rapidity ranges, totaling 20 different fragments of the phase space. In previous sections we have plotted examples of multiplicity distributions and their corresponding combinants. Figures 1 and 3 show the plots for $4 \mathrm{GeV}<p_{T}^{\text {jet }}<6 \mathrm{GeV}$ at $|y|<1.9$ with $R=0.4$, while Figs. 5 and 6 show the same for $10 \mathrm{GeV}<$ $p_{T}^{\text {jet }}<15 \mathrm{GeV}$. Instead of showing all figures for $P(N)$ and $C_{j}$ which we have calculated for each possible $g(N)$ 
TABLE I. Dependence on $p_{T}^{\text {jet }}$ for $R=0.4$ and $0<|y|<1.9$.

\begin{tabular}{lcccccccc}
\hline \hline$p_{T}^{\text {jet }}[\mathrm{GeV}]$ & $\langle N\rangle$ & $\operatorname{Var}(N)$ & \multicolumn{1}{c}{$A$} & \multicolumn{1}{c}{$B$} & \multicolumn{1}{c}{$\alpha$} & \multicolumn{1}{c}{$\delta$} & \multicolumn{1}{c}{$\alpha_{0}$} & $P(0)$ \\
\hline $4-6$ & 3.303 & 1.730 & 13.3 & 10.3 & 14.596 & 1.176 & 0.286 & 0.00430246 \\
$6-10$ & 4.131 & 2.585 & 15.7 & 13.0 & 16.287 & 1.387 & 2.096 & 0.00155689 \\
$10-15$ & 5.170 & 3.946 & 32.7 & 27.9 & 29.374 & 2.220 & 4.367 & 0.000320975 \\
$15-24$ & 6.254 & 5.612 & 127.0 & 125.0 & 126.666 & 3.938 & 6.055 & 0.0000309069 \\
$24-40$ & 7.644 & 7.584 & 196.0 & 180.0 & 179.212 & 7.0 & 7.63 & 0.0000160399 \\
\hline \hline
\end{tabular}

TABLE II. Dependence on $p_{T}^{\text {jet }}$ for $R=0.6$ and $0<|y|<1.9$.

\begin{tabular}{lcrrrrrrl}
\hline \hline$p_{T}^{\text {jet }}[\mathrm{GeV}]$ & $\langle N\rangle$ & $\operatorname{Var}(N)$ & \multicolumn{1}{c}{$A$} & \multicolumn{1}{c}{$B$} & $\alpha$ & $\delta$ & \multicolumn{1}{c}{$\alpha_{0}$} & $P(0)$ \\
\hline $4-6$ & 4.398 & 2.319 & 17.4 & 13.2 & 20.216 & 0.961089 & 0.0 & 0.000767709 \\
$6-10$ & 5.717 & 3.878 & 15.7 & 11.0 & 16.709 & 0.914 & 2.528 & 0.000272948 \\
$10-15$ & 7.374 & 6.878 & 63.2 & 58.0 & 58.875 & 3.167 & 7.209 & 0.0000273138 \\
$15-24$ & 8.526 & 8.420 & 577.7 & 580.0 & 579.390 & 6.168 & 68.509 & 0.00000141011 \\
$24-40$ & 9.790 & 10.775 & 3.3 & 5.9 & 226.418 & -0.00412 & -218.809 & 0.000123035 \\
\hline \hline
\end{tabular}

TABLE III. Dependence on rapidity interval $|y|$ for $R=0.4$ and $10<p_{T}^{\text {jet }}<15 \mathrm{GeV}$.

\begin{tabular}{lcccccccc}
\hline \hline$|y|$ & $\langle N\rangle$ & $\operatorname{Var}(N)$ & $A$ & $B$ & $\alpha$ & $\delta$ & $\alpha_{0}$ & $P(0)$ \\
\hline $0-0.5$ & 5.246 & 3.086 & 24.4 & 27.0 & 27.861 & 2.227 & 4.507 & 0.000330263 \\
$0.5-1$ & 5.178 & 3.949 & 47.3 & 44.0 & 46.500 & 2.631 & 4.483 & 0.000185273 \\
$1-1.5$ & 5.158 & 3.852 & 15.7 & 17.0 & 18.476 & 1.677 & 4.012 & 0.00052248 \\
$1.5-1.9$ & 5.061 & 3.854 & 30.7 & 31.0 & 32.632 & 2.370 & 4.310 & 0.000320779 \\
\hline \hline
\end{tabular}

given by Eq. (20) and $P(N)$ using Eq. (21), we will show parameter values that are there, describing the amplitude of the $C_{j}$ oscillations with the formula

$$
\langle N\rangle\left|C_{j}\right|=A B^{j}
$$

With increasing values of the transverse momenta of jets, $p_{T}^{\text {jet }}$, the mean multiplicity in jets grows and affects the parameters given in the tables. In Table I the parameters depend on $\langle N\rangle$ in the following ways:

$$
\begin{gathered}
\delta=0.94+\left(\frac{\langle N\rangle}{4.9}\right)^{4.4}, \\
\alpha_{0}=-4.94+1.7\langle N\rangle, \\
\alpha \cong A \cong B \cong 10+\left(\frac{\langle N\rangle}{3.05}\right)^{6},
\end{gathered}
$$

$$
P(0)=0.5 \exp \left(-\frac{\langle N\rangle}{0.7}\right)
$$

Table II shows results for broader jets with $R=0.6$. The dependences of the parameters on $\langle N\rangle$ in this case are as follows:

$$
\begin{gathered}
\delta=0.66+\left(\frac{\langle N\rangle}{6.38}\right)^{5.85}, \\
\alpha_{0}=-9.56+2.17\langle N\rangle, \\
\alpha \cong A \cong B \cong 15.5+\left(\frac{\langle N\rangle}{5.9}\right)^{17.5} .
\end{gathered}
$$

The dependence of $P(0)$ on $\langle N\rangle$ remains the same as in Eq. (41).

The value of $\langle N\rangle$ increases by a factor of 1.85 for $R=0.6$ in comparison to the case of $R=0.4$, as seen from a comparison of Tables I and II. Other dependences on $\langle N\rangle$ 
are similar to those from the above equations. However, in the interval of $24 \mathrm{GeV}<p_{T}^{\text {jet }}<40 \mathrm{GeV}$, we have $\delta<0$ and hence this $p_{T}^{\text {jet }}$ interval was omitted in determining the parameter dependence on $\langle N\rangle$.

With regards to the relation between $\operatorname{Var}(N)$ and $\langle N\rangle$, it is observed that for $R=0.4$, we have a dispersion $\sigma=$ $\sqrt{\operatorname{Var}(N)}=0.33\langle N\rangle+0.23$ and for $R=0.6$ we have $\sqrt{\operatorname{Var}(N)}=0.33\langle N\rangle+0.10 .^{1}$

Note that mean multiplicities $\langle N\rangle$ within the various rapidity intervals do not depend significantly on the rapidity interval $|y|$, as shown in Table III, and are similar to $\langle N\rangle$ for the maximal interval $0<|y|<1.9$. Similarly, the other parameters do not differ significantly from the corresponding parameters in the maximum rapidity range (no systematic changes with the width of the rapidity range).

\section{CONCLUSIONS}

The recurrence relation $g(N)=(N+1) P(N+1) / P(N)$ leads to multiplicity distributions of the form

$$
P(N)=\frac{P(0)}{N !} \prod_{i=0}^{N-1} g(i) .
$$

For $g(N)$ given by Eq. (20) with $\alpha_{0}=0$, we have the PD for $\delta=0$, a sub-Poissonian distribution for $\delta>0$ (also known as the Conway-Maxwell-Poisson distribution) [26-30], and a super-Poissonian distribution for $\delta<0$.

However, it turns out that the multiplicity distributions in jets prefer the recurrence relation with $\alpha_{0} \neq 0$, leading to multiplicity distributions of the form given by Eq. (21). For small $\langle N\rangle$ we observe sub-Poissonian distributions with modified combinants oscillating as

$$
\langle N\rangle C_{j} \propto(-1)^{j} \alpha^{j+1} .
$$

The parameters of the multiplicity distributions $\left(\alpha, \alpha_{0}\right.$, and $\delta$ ) depend on $\langle N\rangle$. For large $\langle N\rangle$, distributions are superPoissonian when $-1<\delta<0{ }^{2}$

A sub-Poissonian distribution with $\operatorname{Var}(N)<\langle N\rangle$ has not been very popular in the majority of discussions about multiplicity distributions in high-energy physics so far

\footnotetext{
${ }^{1}$ It is worth recalling at this point that this linear relationship, $\sqrt{\operatorname{Var}(N)}=a\langle N\rangle+b$, known as Wróblewski's law [31], is satisfied for a wide range of multiparticle production processes, like $p p$ collisions (here, $a=-b=0.585$ ), both $\pi^{+} p$ and $\pi^{-} p$ collisions (with $a=0.44$ for $\pi^{ \pm}, b=-0.22$ for $\pi^{+}$, and $b=$ -0.9 for $\pi^{-}$) [32], and $e^{+} e^{-}$collisions (where $a=0.25$ and $b=0.7)$ [33].

${ }^{2}$ For $\delta=-1$ we have the well-known NBD, for $\alpha_{0}>\alpha>0$ (where the parameter $k=\alpha_{0} / \alpha+1$, if additionally $\alpha_{0}=0$ ) we would have a geometric distribution, the widest one), and for $\alpha_{0}>0$ and $\alpha<0$ we would have the BD (where the parameter $\left.K=-\alpha_{0} / \alpha-1\right)$.
}

(mainly due to the fact that this phenomenon is only observed when $\langle N\rangle$ is not too large). However, it is quite an important distribution [17,34]. The existence of a subPoissonian distribution implies at least one of the following scenarios: 1) the underlying elementary processes are not totally random (partially deterministic), or 2) the classical Markov processes describing them require further generalizations. Either conclusion forces us to modify the successful stochastic approach. In our case, we have shown that the sub-Poissonian multiplicity distributions describing the experimental data can be naturally interpreted as stochastic Markov processes in which the birth and death rates are both multiplicity dependent.

\section{ACKNOWLEDGMENTS}

This research was supported in part by the National Science Centre, Poland (NCN) Grants No. 2020/39/O/ST2/ 00277 (M. R.) and No. 2016/22/M/ST2/00176 (G. W.) and by the Polish Ministry of Science and Higher Education Grant No. DIR/WK/2016/2018/17-1 (G. W.). H. W. A. would like to thank the NUS Research Scholarship for supporting this study. In preparation for this work we used the resources of the Center for Computation and Computational Modeling of the Faculty of Exact and Natural Sciences of the Jan Kochanowski University in Kielce.

\section{APPENDIX A: RELATIONSHIP OF COMBINANTS WITH FACTORIAL MOMENTS AND CUMULANTS}

Usually information contained in $P(N)$ is obtained by examining their corresponding factorial moments, $F_{q}$, and cumulant factorial moments, $K_{q}$, (or their ratios) (cf. Refs. [1,2]),

$$
K_{q}=F_{q}-\sum_{i=1}^{q-1}\left(\begin{array}{c}
q-1 \\
i-1
\end{array}\right) K_{q-i} F_{i},
$$

where

$$
F_{q}=\sum_{N=q}^{\infty} N(N-1)(N-2) \ldots(N-q+1) P(N)
$$

are the factorial moments. As shown in Refs. [9,11], the $K_{q}$ can be expressed as an infinite series of the $C_{j}$,

$$
K_{q}=\sum_{j=q}^{\infty} \frac{(j-1) !}{(j-q) !}\langle N\rangle C_{j-1}
$$

and, conversely, the $C_{j}$ can be expressed in terms of the $K_{q}[1,2]$, 


$$
C_{j}=\frac{1}{\langle N\rangle} \frac{1}{(j-1) !} \sum_{p=0}^{\infty} \frac{(-1)^{p}}{p !} K_{p+j} .
$$

Note that $C_{j}$ depend only on multiplicities smaller than their rank [14,15], while the moments $K_{q}$ require knowledge of all $P(N)$ and therefore are very sensitive to possible limitations of the available phase space [1,2]. On the other hand, calculations of combinants require knowledge of $P(0)$, which may not always be available. Both $C_{j}$ and $K_{q}$ exhibit the property of additivity.

\section{APPENDIX B: MULTIPLICITIES FROM TWO BINOMIAL DISTRIBUTIONS}

If we have two sources producing $N_{1}$ and $N_{2}$ particles, respectively, and they are distributed according to a BD defined by the parameters $\left(K_{1}, p_{1}\right)$ and $\left(K_{2}, p_{2}\right)$, then the distribution of $N=N_{1}+N_{2}$ particles,

$$
P(N)=\sum_{i=0}^{\min \left(N, K_{1}, K_{2}\right)} P_{1}(i) P_{2}(N-i),
$$

is described by a generating function comprising the product of generating functions for both sources, i.e.,

$$
G(z)=\left(1-p_{1}+p_{1} z\right)^{K_{1}} \cdot\left(1-p_{2}+p_{2} z\right)^{K_{2}} .
$$

In this case, the first two moments of the distribution $P(N)$ are given by

$$
\begin{aligned}
\langle N\rangle & =\left.\frac{d G(z)}{d z}\right|_{z=1}=K_{1} p_{1}+K_{2} p_{2}, \\
\operatorname{Var}(N) & =\left.\frac{d^{2} G(z)}{d z^{2}}\right|_{z=1}+\langle N\rangle-\langle N\rangle^{2} \\
& =K_{1} p_{1}\left(1-p_{1}\right)+K_{2} p_{2}\left(1-p_{2}\right) .
\end{aligned}
$$

Denoting the modified combinant of the first and second BD components as $C(1)_{j}$ and $C(2)_{j}$ respectively, the overall modified combinant $\langle N\rangle C_{j}$ can be written as

$$
\begin{aligned}
\langle N\rangle C_{j} & =\left\langle N_{1}\right\rangle C(1)_{j}+\left\langle N_{2}\right\rangle C(2)_{j} \\
& =(-1)^{j}\left\{K_{1}\left[\frac{p_{1}}{\left(1-p_{1}\right)}\right]^{j+1}+K_{2}\left[\frac{p_{2}}{\left(1-p_{2}\right)}\right]^{j+1}\right\} .
\end{aligned}
$$

The value of one of the $p$ parameters must be carefully chosen to reflect the observed increase in the amplitude of

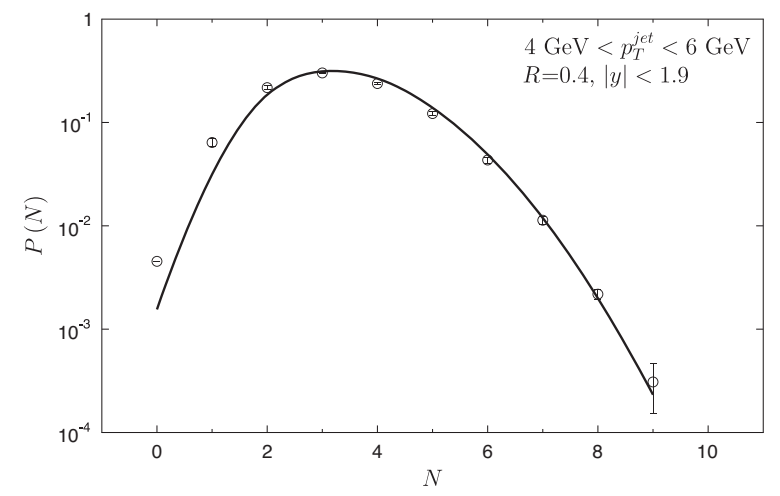

FIG. 7. Points: $P(N)$ from ATLAS data for jets with $4 \mathrm{GeV}<$ $p_{T}^{\text {jet }}<6 \mathrm{GeV}$ and radius parameter $R=0.4$, over the full measured rapidity range $|y|<1.9$. The curve fitting these data comes from the generating function given by Eq. (B2).

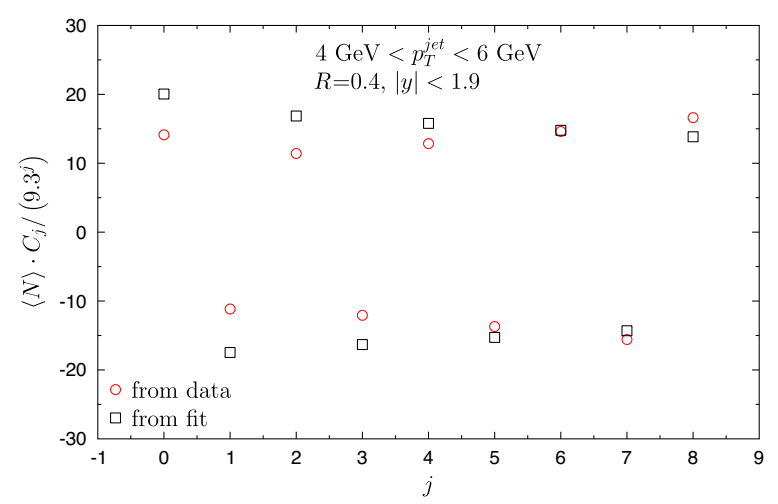

FIG. 8. Comparison of $C_{j}$ for multiplicity distributions from Fig. 7. Red circles: $C_{j}$ from data on $P(N)$, black squares: $C_{j}$ from Eq. (B4) obtained from theoretical $P(N)$ defined by the generating function given by Eq. (B2).

$C_{j}$. Setting (indicative) parameter values $K_{1}=2, p_{1}=0.9$ and $K_{2}=10, p_{2}=0.17$, we have $P(N)$ and $C_{j}$ as shown in Figs. 7 and 8. For this set of parameters used to fit data, we have $\langle N\rangle=3.5$ and $\operatorname{Var}(N)=1.6$.

While it is possible to reasonably describe either $P(N)$ or $C_{j}$ with a suitable choice of parameters, it is not yet possible to describe both simultaneously. Figures 7 and 8 show the extent of the deviation of such an approach from data.

It turns out that while by suitable choice of parameters, we can describe (more or less reasonably) separately $P(N)$ or $C_{j}$, but not simultaneously both observables. Figures 7 and 8 demonstrate to what extent is it possible to get closer to this goal in such an approach. 
[1] W. Kittel and E. A. De Wolf, Soft Multihadron Dynamics (World Scientific, Singapore, 2005).

[2] R. Botet and M. Płoszajczak, Universal Fluctuations, The Phenomenology of Hadronic Matter (World Scientific, Singapore, 2002).

[3] T. F Hoang and B. Cork, Z. Phys. C 36, 323 (1987).

[4] S. V. Chekanov and V. I. Kuvshinow, J. Phys. G 22, 601 (1996).

[5] I. Zborovsky, arXiv:1106.4697.

[6] B. E. A. Saleh and M. K. Teich, Proc. IEEE 70, 229 (1982).

[7] G. Wilk and Z. Włodarczyk, J. Phys. G 44, 015002 (2017).

[8] G. Wilk and Z. Włodarczyk, Int. J. Mod. Phys. A 33, 1830008 (2018).

[9] M. Rybczyński, G. Wilk, and Z. Włodarczyk, Phys. Rev. D 99, 094045 (2019).

[10] H. W. Ang, M. Ghaffar, A. H. Chan, M. Rybczyński, Z. Włodarczyk, and G. Wilk, Mod. Phys. Lett. A 34, 1950324 (2019).

[11] H. W. Ang, A. H. Chan, M. Ghaffar, M. Rybczyński, G. Wilk, and Z. Włodarczyk, Eur. Phys. J. A 56, 117 (2020).

[12] G. Wilk and Z. Włodarczyk, Int. J. Mod. Phys. A 36, 2150072 (2021).

[13] I. Zborovsky, Eur. Phys. J. C 78, 816 (2018).

[14] S. K. Kauffmann and M. Gyulassy, J. Phys. A 11, 1715 (1978).

[15] M. Gyulassy and S. K. Kauffman, Phys. Rev. Lett. 40, 298 (1978).

[16] R. Vasuvedan, P. R. Vitall, and K. V. Parthasarathy, J. Phys. A 17, 989 (1984).

[17] P. Carruthers and C. C. Shih, Int. J. Mod. Phys. A 02, 1447 (1987).
[18] A. B. Balantekin and J. E. Seger, Phys. Lett. B 266, 231 (1991).

[19] I. Szapudi and A. S. Szalay, Astrophys. J. 408, 43 (1993).

[20] S. J. Lee and A.Z. Mekjian, Nucl. Phys. A730, 514 (2004).

[21] A. Z. Mekjian, T. Csörgö, and S. Hegyi, Nucl. Phys. A784, 515 (2007).

[22] G. Aad et al. (ATLAS Collaboration), Phys. Rev. D 84, 054001 (2011).

[23] Durham HepData Project: https://www.hepdata.net/record/ 57743 ?.

[24] G. Wilk and Z. Włodarczyk, Phys. Lett. B 727, 163 (2013).

[25] N. G. Watson, A Treatise on the Theory of Bessel Functions, 2nd ed. (Cambridge University Press, Cambridge, England, 1944), pp. 500-501.

[26] R. W. Conway and W. L. Maxwell, J. Ind. Eng. 12, 132 (1962).

[27] G. Shmueli, T. P. Minka, J. B. Kadane, S. Borle, and P. Boatwright, J. R. Stat. Soc. C Appl. Stat. 54, 127 (2005).

[28] S. Chakraborty and T. Imoto, J. Stat. Distrib. Appl. 3, 5 (2016).

[29] S. B. Chatla and G. Shmueli, Comput. Stat. Data Anal. 121, 71 (2018).

[30] B. Li, H. Zhang, and J. He, Comm. Stat. Theor. Meth. 49, 1311 (2020).

[31] A. K. Wróblewski, Acta Phys. Pol. B 4, 857 (1973).

[32] L. Van Hove, Phys. Lett. 43B, 65 (1973).

[33] R. Szwed, G. Wrochna, and A. K. Wróblewski, Mod. Phys. Lett. A 05, 1851 (1990).

[34] C. C. Shih, Phys. Rev. D 34, 2720 (1986). 